\title{
Nonlocal problems for first order functional partial differential equations
}

\author{
by JAN TURo (Gdańsk)
}

\begin{abstract}
Local existence of generalized solutions to nonlocal problems for nonlinear functional partial differential equations of first order is investigated. The proof is based on the bicharacteristics and successive approximations methods.
\end{abstract}

1. Introduction. Let $B=\left[-b_{0}, 0\right] \times[-b, b], I_{t}=[0, t] \times \mathbb{R}^{n}$ and $E_{t}=$ $\left[-b_{0}, t\right] \times \mathbb{R}^{n}$, where $t \in[0, a), a>0, b=\left(b_{1}, \ldots, b_{n}\right) \in \mathbb{R}_{+}^{n}, \mathbb{R}_{+}=[0, \infty)$, and $b_{0} \in \mathbb{R}_{+}$. For any function $z: E_{a} \rightarrow \mathbb{R}$ with $a>0$ and a fixed $(x, y)=$ $\left(x, y_{1}, \ldots, y_{n}\right) \in I_{a}$ we define the function $z_{(x, y)}: B \rightarrow \mathbb{R}$ by $z_{(x, y)}(t, s)=$ $z(x+t, y+s),(t, s) \in B$. We denote by $C^{0}(B, \mathbb{R})$ the set of all continuous real functions on $B$.

We assume that $f: I_{a} \times C^{0}(B, \mathbb{R}) \times \mathbb{R}^{n} \rightarrow \mathbb{R}$, and $h_{l}, \varphi: E_{0}=\left[-b_{0}\right] \times$ $\mathbb{R}^{n} \rightarrow \mathbb{R}, l=1, \ldots, r$, are given functions.

We consider the nonlinear functional partial differential equation

$$
D_{x} z(x, y)=f\left(x, y, z_{(x, y)}, D_{y} z(x, y)\right)
$$

with nonlocal condition

$$
z(x, y)+\sum_{l=1}^{r} h_{l}(x, y) z\left(a_{k}+x, y\right)=\varphi(x, y), \quad(x, y) \in E_{0},
$$

where $D_{y} z=\left(D_{y_{1}} z, \ldots, D_{y_{n}} z\right)$, and $a_{l}, i=1, \ldots, r$, are numbers such that $0<a_{1}<\ldots<a_{r} \leq a$.

The nonlocal condition (2) may also be written in the form

$$
z_{(0, y)}+\sum_{l=1}^{r}\left(h_{l}\right)_{(0, y)} z_{\left(a_{l}, y\right)}=\varphi, \quad y \in \mathbb{R}^{n} .
$$

1991 Mathematics Subject Classification: 35L50, 35R10.

Key words and phrases: nonlocal problem, differential-functional equation, generalized solution. 
In this paper we extend the method of quasilinearization to problem (1), (2), for which we construct two uniformly convergent sequences: the first converges to a solution of the problem, the second to its derivative. The technique essentially involves the method of bicharacteristics.

We consider generalized solutions of problem (1), (2). More precisely, a function $u: E_{c} \rightarrow \mathbb{R}$, where $0<c \leq a$, is a solution of (1),(2) provided

(i) $u \in C\left(E_{c}, \mathbb{R}\right)$ and $D_{y} u(x, y)$ exists for $(x, y) \in I_{c}$;

(ii) the function $u(\cdot, y):[0, c] \rightarrow \mathbb{R}$ is absolutely continuous on $[0, c]$ for each $y \in \mathbb{R}^{n}$;

(iii) for each $y \in \mathbb{R}^{n}$ equation (1) is satisfied for almost all $x \in[0, c]$, and condition (2) holds.

For $r=n, b_{0}=0$ and $h_{l i j}=h_{i j} \delta_{l i}$ ( $\delta_{l i}$ is the Kronecker symbol) the nonlocal boundary condition (2) reduces to the nonlocal condition "à la Cesari" [1]-[4], [11], [12], [20], [26], [27]. If $h_{l i j}=\delta_{l i} \delta_{i j}$ then (2) reduces to the Nicoletti condition [21], [23]. Furthermore, if all $a_{l}=0, l=1, \ldots, r$, then we get the usual Cauchy condition.

Classical solutions to nonlocal problems were considered for parabolic equations in [8], [9], [13], and for hyperbolic equations in [6], [7], [10], [15].

Generalized solutions to nonlinear first order partial differential equations have been investigated in a large number of papers by various authors. Theorems on existence, uniqueness and continuous dependence upon Cauchy or boundary data for quasilinear systems were given in [1]-[3], [11], [12], [16], [17], [22]. Quasilinear differential-integral systems and systems with a retarded argument were considered in [4], [19], [20]. Nonlinear differential equations were studied in [14]. Generalized solutions of quasilinear or nonlinear equations with operators of the Volterra type were investigated in [24]-[27]. Generalized solutions of (1) with the Cauchy condition were considered in [5].

Equation (1) contains as particular cases the differential equations with a retarded argument, differential-integral equations and differential-functional equations with operators of the Volterra type (see Section 6).

2. Preliminaries and assumptions. For $y \in \mathbb{R}^{n}$ we write $\|y\|=$ $\sum_{i=1}^{n}\left|y_{i}\right|$. Let $\|\cdot\|_{0}$ denote the supremum norm in $C^{0}(B, \mathbb{R})$. We denote by $C^{0, \bar{L}}(B, \mathbb{R})$ the class of all functions $w \in C^{0}(B, \mathbb{R})$ such that

$$
\|w\|_{L}=\sup \left\{|w(t, s)-w(t, \bar{s})| \cdot\|s-\bar{s}\|^{-1}:(t, s),(t, \bar{s}) \in B\right\}<\infty .
$$

For $w \in C^{0, L}(B, \mathbb{R})$ we put $\|w\|_{0, L}=\|w\|_{0}+\|w\|_{L}$.

Let $C^{1}(B, \mathbb{R})$ be the set of all continuous functions $w: B \rightarrow \mathbb{R}$ such that the derivatives $D_{s} w=\left(D_{s_{1}} w, \ldots, D_{s_{n}} w\right)$ exist and $D_{s} w \in C^{0}\left(B, \mathbb{R}^{n}\right)$. For $w \in C^{1}(B, \mathbb{R})$ we put $\|w\|_{1}=\|w\|_{0}+\max \left\{\left\|D_{s} w(t, s)\right\|:(t, s) \in B\right\}$. 
We denote by $C^{1, L}(B, \mathbb{R})$ the set of all functions $w \in C^{1}(B, \mathbb{R})$ such that $D_{s} w \in C^{0, L}(B, \mathbb{R})$. For $w \in C^{1, L}(B, \mathbb{R})$ we write $\|w\|_{1, L}=\|w\|_{1}+$ $\left\|D_{s} w\right\|_{L}$.

Let $\Theta$ be the class of all functions $\gamma:[0, a] \times \mathbb{R}_{+} \rightarrow \mathbb{R}_{+}$such that $\gamma(\cdot, t) \in$ $L\left([0, a], \mathbb{R}_{+}\right)$for every $t \in \mathbb{R}_{+}$, and $\gamma(s, \cdot): \mathbb{R}_{+} \rightarrow \mathbb{R}_{+}$is nondecreasing for almost all $s \in[0, a]$, where $L\left([0, a], \mathbb{R}_{+}\right)$is the class of all integrable functions on $[0, a]$.

We put $\Omega^{(i)}=I_{a} \times C^{i}(B, \mathbb{R}) \times \mathbb{R}^{n}$ for $i=1,2$, and $\Omega^{(1, L)}=I_{a} \times$ $C^{1, L}(B, \mathbb{R}) \times \mathbb{R}^{n}$.

Given $S=\left(S_{0}, S_{1}, S_{2}\right) \in \mathbb{R}_{+}^{3}$, we denote by $C^{1, L}[S]$ the set of all functions $\varphi: E_{0} \rightarrow \mathbb{R}$ such that

(i) $\varphi \in C\left(E_{0}, \mathbb{R}\right)$ and $D_{y} \varphi(x, y)$ exists for $(x, y) \in E_{0}$;

(ii) $|\varphi(x, y)| \leq S_{0},\left\|D_{y} \varphi(x, y)\right\| \leq S_{1}$ on $E_{0}$, and

$$
\left\|D_{y} \varphi(x, y)-D_{y} \varphi(x, \bar{y})\right\| \leq S_{2}\|y-\bar{y}\|
$$

for $(x, y),(x, \bar{y}) \in E_{0}$.

Given $\bar{S}=\left(\bar{S}_{0}, \bar{S}_{1}, \bar{S}_{2}\right) \in \mathbb{R}_{+}^{3}, \bar{S}_{0}, \bar{S}_{1} \in[0,1 / 2)$, we denote by $C^{1, L}[\bar{S}]$ the set of all functions $h_{l}: E_{0} \rightarrow \mathbb{R}$ such that

(i) $h_{l} \in C\left(E_{0}, \mathbb{R}\right)$ and $D_{y} h_{l}(x, y)$ exists for $(x, y) \in E_{0}, l=1, \ldots, r$;

(ii) $\sum_{l=1}^{r}\left|h_{l}(x, y)\right| \leq \bar{S}_{0}, \sum_{l=1}^{r}\left\|D_{y} h_{l}(x, y)\right\| \leq \bar{S}_{1}$ on $E_{0}$, and

$$
\sum_{l=1}^{r}\left\|D_{y} h_{l}(x, y)-D_{y} h_{l}(x, \bar{y})\right\| \leq S_{2}\|y-\bar{y}\|
$$

for $(x, y),(x, \bar{y}) \in E_{0}$.

Let $\varphi \in C^{1, L}[S], h_{l} \in C^{1, L}[\bar{S}], l=1, \ldots, r$, be given and let $0<c \leq a$, $Q=\left(Q_{0}, Q_{1}, Q_{2}\right), \lambda=\left(\lambda_{0}, \lambda_{1}\right)$, where $Q_{i} \in \mathbb{R}_{+}, i=0,1,2$, and $\lambda_{j} \in$ $L\left([0, a], \mathbb{R}_{+}\right), j=0,1$. We denote by $C^{1, L}[c, \varphi, h, Q, \lambda]$ the set of all functions $z \in C\left(E_{c}, \mathbb{R}\right)$ such that

(i) $z$ satisfies condition $(2)$ on $E_{0}$;

(ii) $D_{y} z(x, y)$ exists for $(x, y) \in I_{c}$;

(iii) $|z(x, y)| \leq Q_{0}$ and $\left\|D_{y} z(x, y)\right\| \leq Q_{1}$ for $(x, y) \in I_{c}$;

(iv) for $(x, y),(\bar{x}, y),(\bar{x}, \bar{y}) \in I_{c}$ we have

$$
\begin{aligned}
|z(x, y)-z(\bar{x}, y)| & \leq\left|\int_{x}^{\bar{x}} \lambda_{0}(t) d t\right| \\
\left\|D_{y} z(x, y)-D_{y} z(\bar{x}, \bar{y})\right\| & \leq\left|\int_{x}^{\bar{x}} \lambda_{1}(t) d t\right|+Q_{2}\|y-\bar{y}\| .
\end{aligned}
$$
that

We denote by $C^{0, L}\left[c, Q, \lambda_{1}\right]$ the class of all functions $v \in C\left(I_{c}, \mathbb{R}^{n}\right)$ such 
(i) $\|v(x, y)\| \leq Q_{1}$ for $(x, y) \in I_{c}$;

(ii) $\|v(x, y)-v(\bar{x}, \bar{y})\| \leq\left|\int_{x}^{\bar{x}} \lambda_{1}(t) d t\right|+Q_{2}\|y-\bar{y}\|$ for $(x, y),(\bar{x}, \bar{y}) \in I_{c}$.

Let $C L(B, \mathbb{R})$ denote the set of all continuous linear operators defined on $C(B, \mathbb{R})$ and taking values in $\mathbb{R}$. The norm in $C L(B, \mathbb{R})$ is denoted by $\|\cdot\|_{C L(B, \mathbb{R})}$.

Assumption $\mathrm{H}_{1}$. $1^{\circ}$ The function $f(\cdot, y, w, q):[0, a] \rightarrow \mathbb{R}$ is measurable for every $(y, w, q) \in \mathbb{R}^{n} \times C^{0}(B, \mathbb{R}) \times \mathbb{R}^{n}$ and $f(x, \cdot): \mathbb{R}^{n} \times C^{0}(B, \mathbb{R}) \times \mathbb{R}^{n} \rightarrow \mathbb{R}$ is continuous for a.e. $x \in[0, a]$;

$2^{\circ}$ there is $\beta \in \Theta$ such that $|f(x, y, w, q)| \leq \beta\left(x,\|w\|_{0}\right)$ on $\Omega^{(0)}$;

$3^{\circ}$ for every $(x, y, w, q) \in \Omega^{(1)}, D_{y} f(x, y, w, q) \in \mathbb{R}^{n}$ and $D_{w} f(x, y, w, q)$ $\in C L(B, \mathbb{R})$ exist;

$4^{\circ}$ there is $\alpha \in \Theta$ such that

$\left\|D_{y} f(x, y, w, q)\right\| \leq \alpha\left(x,\|w\|_{1}\right), \quad\left\|D_{w} f(x, y, w, q)\right\|_{C L(B, \mathbb{R})} \leq \alpha\left(x,\|w\|_{1}\right)$, on $\Omega^{(1)}$;

$5^{\circ}$ there is $\gamma \in \Theta$ such that

$$
\begin{aligned}
& \left\|D_{y} f(x, y, w, q)-D_{y} f(x, \bar{y}, w+h, \bar{q})\right\| \\
& \quad \leq \gamma\left(x,\|w\|_{1, L}\right)\left[\|y-\bar{y}\|+\|h\|_{1}+\|q-\bar{q}\|\right],
\end{aligned}
$$

and

$$
\begin{aligned}
\left\|D_{w} f(x, y, w, q)-D_{w} f(x, \bar{y}, w+h, \bar{q})\right\|_{C L(B, \mathbb{R})} & \\
& \leq \gamma\left(x,\|w\|_{1, L}\right)\left[\|y-\bar{y}\|+\|h\|_{1}+\|q-\bar{q}\|\right],
\end{aligned}
$$

for $(x, y, w, q) \in \Omega^{(1, L)}, \bar{y}, \bar{q} \in \mathbb{R}^{n}, h \in C^{1}(B, \mathbb{R})$.

Assumption $\mathrm{H}_{2}$. $1^{\circ} D_{q} f$ exists for every $(x, y, w, q) \in \Omega^{(1)}$ and $D_{q} f(\cdot, y, w, q) \in L\left([0, a], \mathbb{R}^{n}\right)$ for $(y, w, q) \in \mathbb{R}^{n} \times C^{1}(B, \mathbb{R}) \times \mathbb{R}^{n}$;

$2^{\circ}$ for $(x, y, w, q) \in \Omega^{(1)}$ we have the estimate

$$
\left\|D_{q} f(x, y, w, q)\right\| \leq \alpha\left(x,\|w\|_{1}\right)
$$

$3^{\circ}$ for $(x, y, w, q) \in \Omega^{(1, L)}, \bar{y}, \bar{q} \in \mathbb{R}^{n}$ and $h \in C^{1}(B, \mathbb{R})$ we have the estimate

$$
\begin{aligned}
& \left\|D_{q} f(x, y, w, q)-D_{q} f(x, \bar{y}, w+h, \bar{q})\right\| \\
& \quad \leq \gamma\left(x,\|w\|_{1, L}\right)\left[\|y-\bar{y}\|+\|h\|_{1}+\|q-\bar{q}\|\right],
\end{aligned}
$$

REMARK 1. We prove the theorem on the existence of solutions and we give an estimate of the domain of solutions. For simplicity of the estimates of the constant $c$ we have assumed the same estimate for the derivatives $D_{y} f, D_{w} f, D_{q} f$, and we have also assumed the Lipschitz condition for these derivatives with the same constant.

REMARK 2. It is important in our considerations that the Lipschitz conditions for the given functions are local with respect to the functional variable. 
Consider the function $D_{y} f$ only and the simplest assumptions on it. Suppose that there is a constant $\widetilde{\gamma}$ such that

(5) $\quad\left\|D_{y} f(x, y, w, q)-D_{y} f(x, \bar{y}, w+h, \bar{q})\right\| \leq \widetilde{\gamma}\left[\|y-\bar{y}\|+\|h\|_{1}+\|q-\bar{q}\|\right]$.

Of course, our results are true if we assume (5) instead of (4).

Now we show that there is a class of nonlinear equations satisfying (4) and not satisfying (5).

Consider the equation with a deviated argument

$$
D_{x} z(x, y)=\widetilde{f}\left(x, y, z(\lambda(x), \psi(x, y)), D_{y} z(x, y)\right),
$$

where $\tilde{f}: I_{a} \times \mathbb{R} \times \mathbb{R}^{n} \rightarrow \mathbb{R}, \quad \lambda \in C([0, a], \mathbb{R}), \quad \psi \in C\left(I_{a}, \mathbb{R}^{n}\right), \quad-b_{0} \leq$ $\lambda(x)-x \leq 0,-b \leq \psi(x, y)-y \leq b,(x, y) \in I_{a}$, and the derivative $D_{y} \psi$ exists on $I_{a}$. We get equation (6) by putting in (1)

$$
f(x, y, w, q)=\widetilde{f}(x, y, w(\lambda(x)-x, \psi(x, y)-y), q) .
$$

Suppose that there are constants $\alpha_{1}, \gamma_{1}, \delta_{0}, \delta_{1} \in \mathbb{R}_{+}$such that

$$
\begin{gathered}
\left\|D_{y} \widetilde{f}(x, y, w, q)-D_{y} \widetilde{f}(x, \bar{y}, \bar{w}, \bar{q})\right\| \leq \gamma_{1}[\|y-\bar{y}\|+|w-\bar{w}|+\|q-\bar{q}\|], \\
\left\|D_{w} \widetilde{f}(x, y, w, q)-D_{w} \widetilde{f}(x, \bar{y}, \bar{w}, \bar{q})\right\| \leq \gamma_{1}[\|y-\bar{y}\|+|w-\bar{w}|+\|q-\bar{q}\|], \\
\left\|D_{w} \widetilde{f}(x, y, w, q)\right\| \leq \alpha_{1}, \quad\left\|D_{y} \psi(x, y)\right\| \leq \delta_{0}, \\
\left\|D_{y} \psi(x, y)-D_{y} \psi(x, \bar{y})\right\| \leq \delta_{1}\|y-\bar{y}\| .
\end{gathered}
$$

Observe that in $\Omega^{(1)}$ we have

$$
\begin{aligned}
D_{y_{i}} f(x, y, w, q)= & D_{y_{i}} \widetilde{f}(x, y, w(\lambda(x)-x, \psi(x, y)-y), q) \\
& +D_{w} \widetilde{f}(x, y, w(\lambda(x)-x, \psi(x, y)-y), q) \\
& \cdot\left[\sum_{j=1}^{n} D_{y_{j}} w(\lambda(x)-x, \psi(x, y)-y)\left(D_{y_{i}} \psi_{j}(x, y)-1\right)\right] .
\end{aligned}
$$

Then it is easy to see that

$$
\begin{aligned}
\left\|D_{y} f(x, y, w, q)-D_{y} f(x, \bar{y}, w+h, \bar{q})\right\| \\
\leq\{ \\
\left.\quad \times \gamma_{1}\left[1+\left(1+\delta_{0}\right)\|w\|_{1}\right]^{2}+\alpha_{1}\left[\left(1+\delta_{0}\right)^{2}\|w\|_{1, L}+\delta_{1}\|w\|_{1}\right]\right\} \\
\quad \times\left[\|y-\bar{y}\|+\|h\|_{1}+\|q-\bar{q}\|\right] .
\end{aligned}
$$

Thus condition (4) is satisfied. We see at once that the function $D_{y} f$ does not satisfy the global Lipschitz condition (5). Similar considerations apply to $D_{w} f, D_{q} f$.

3. Bicharacteristics. Suppose that $h_{l} \in C^{1, L}[\bar{S}], l=1, \ldots, r, \varphi \in$ $C^{1, L}[S], z \in C^{1, L}[c, \varphi, h, Q, \lambda]$ and $u \in C^{0, L}\left[c, Q, \lambda_{1}\right]$. We consider the Cauchy problem

$$
\eta^{\prime}(t)=-D_{q} f\left(t, \eta(t), z_{(t, \eta(t))}, u(t, \eta(t))\right), \quad \eta(x)=y,
$$

and denote by $g[z, u](\cdot, x, y)$ its solution. 
By using the Gronwall inequality, we can prove, similarly to [5], [28], the following.

Lemma 1. Let $\varphi, \bar{\varphi} \in C^{1, L}[S], h_{l}, \bar{h}_{l} \in C^{1, L}[\bar{S}], z \in C^{1, L}[c, \varphi, h, Q, \lambda]$, $\bar{z} \in C^{1, L}[c, \bar{\varphi}, \bar{h}, Q, \lambda], u, \bar{u} \in C^{0, L}\left[c, Q, \lambda_{1}\right]$ be given, and let Assumption $\mathrm{H}_{2}$ be satisfied. Then the solutions $g[z, u](\cdot ; x, y)$ and $g[\bar{z}, \bar{u}](\cdot, x, y)$ of $(7)$ exist on $[0, c]$, they are unique and we have the estimates

$$
\|g[z, u](t ; x, y)-g[z, u](t ; \bar{x}, \bar{y})\| \leq G(x)\left[\left|\int_{x}^{\bar{x}} \alpha\left(s, Q^{\prime}\right) d s\right|+\|y-\bar{y}\|\right]
$$

and

$$
\begin{aligned}
\|g[z, u](t ; x, y)-g[\bar{z}, \bar{u}](t ; x, y)\| & \\
\leq & G(x) \mid \int_{x}^{\bar{t}} \gamma\left(s, Q^{*}\right) d s\left[\|z-\bar{z}\|_{C\left(E_{s}, \mathbb{R}\right)}\right. \\
& \left.\quad+\left\|D_{y} z-D_{y} \bar{z}\right\|_{C\left(E_{s}, \mathbb{R}^{n}\right)}+\|u-\bar{u}\|_{C\left(I_{s}, \mathbb{R}^{n}\right)}\right] \mid,
\end{aligned}
$$

where

$$
G(x)=\exp \left[\bar{Q} \int_{0}^{x} \gamma\left(s, Q^{*}\right) d s\right],
$$

and $Q^{\prime}=Q_{0}+Q_{1}, \bar{Q}=1+Q_{1}+2 Q_{2}, Q^{*}=Q_{0}+Q_{1}+Q_{2}$.

For given $\varphi \in C^{1, L}[S], h_{l} \in C^{1, L}[\bar{S}], z \in C^{1, L}[c, \varphi, h, Q, \lambda]$ and $u \in$ $C^{0, L}\left[c, Q, \lambda_{1}\right]$, we define

$$
\begin{aligned}
F[z, u](x, y)= & \varphi(0, g(0 ; x, y))-\sum_{l=1}^{r} h_{l}(0, g(0 ; x, y)) z\left(a_{l}, g(0 ; x, y)\right) \\
& +\int_{0}^{x}[f(P[z, u](s ; x, y)) \\
& \left.-\sum_{k=1}^{n} D_{q_{k}} f(P[z, u](s ; x, y)) u_{k}(s, g(s ; x, y))\right] d s
\end{aligned}
$$

and

$$
\begin{aligned}
T_{i}[z, u](x, y)= & D_{y_{i}} \varphi(0, g(0 ; x, y))-\sum_{l=1}^{r} D_{y_{i}} h_{l}(0, g(0 ; x, y)) z\left(a_{l}, g(0 ; x, y)\right) \\
& -\sum_{l=1}^{r} h_{l}(0, g(0 ; x, y)) u\left(a_{l}, g(0 ; x, y)\right) \\
& +\int_{0}^{x}\left[D_{y_{i}} f(P[z, u](s ; x, y))\right. \\
& \left.+D_{w} f(P[z, u](s ; x, y))\left(u_{i}\right)_{(s, g(s ; x, y))}\right] d s,
\end{aligned}
$$


where

$$
P[z, u](\tau ; x, y)=\left(\tau, g[z, u](\tau ; x, y), z_{(\tau, g[z, u](\tau ; x, y))}, u(\tau, g[z, u](\tau ; x, y))\right),
$$

and $i=1, \ldots, n$.

4. Successive approximations. Now we consider the system of integral-functional equations which are generated by (1), (2):

$$
\begin{aligned}
& z(x, y)=F[z, u](x, y), \quad u(x, y)=T[z, u](x, y), \quad(x, y) \in I_{a}, \\
& z(x, y)+\sum_{l=1}^{r} h_{l}(x, y) z\left(a_{k}+x, y\right)=\varphi(x, y), \quad(x, y) \in E_{0}, \\
& g(t ; x, y)=y+\int_{t}^{x} D_{q} f(P[z, u](s ; x, y)) d s,
\end{aligned}
$$

where $T=\left(T_{1}, \ldots, T_{n}\right)$.

REMARK 3. The functional integral system (8) is obtained in the following way. By introducing an additional unknown function $u=D_{y} z$ in (1) we consider the linearization of this equation with respect to $u$, i.e.

$$
D_{x} z(x, y)=f(V)+\sum_{j=1}^{n} D_{q_{j}} f(V)\left(D_{y_{j}} z(x, y)-u_{j}(x, y)\right),
$$

where $V=\left(x, y, z_{(x, y)}, u(x, y)\right)$. From (1) we get the following differential system for the unknown function $u$ :

$$
D_{x} u_{i}(x, y)=D_{y_{i}} f(V)+D_{w} f(V)\left(D_{y_{i}} z\right)_{(x, y)}+\sum_{j=1}^{n} D_{q_{j}} f(V) u_{i}(x, y),
$$

$i=1, \ldots, n$. Finally, we put $D_{y} z=u$ in (10).

Considering (9), (10) along the bicharacteristic $g[z, u](\cdot ; x, y)$, we obtain

$$
\begin{aligned}
\frac{d}{d t} z(t, g[z, u](t ; x, y))= & f(P[z, u](t ; x, y)) \\
& -\sum_{j=1}^{n} D_{q_{j}} f(P[z, u](t ; x, y)) u_{j}(t, g[z, u](t ; x, y)), \\
\frac{d}{d t} u_{i}(t, g[z, u](t ; x, y))= & D_{y_{i}} f(P[z, u](t ; x, y)) \\
& +D_{w} f(P[z, u](t ; x, y))\left(u_{i}\right)_{(t, g[z, u](t ; x, y))} .
\end{aligned}
$$

Now we construct successive approximations. Suppose that $\varphi \in C^{1, L}[S]$, $h_{l} \in C^{1, L}[\bar{S}], l=1, \ldots, r$, and that Assumptions $\mathrm{H}_{1}-\mathrm{H}_{2}$ are satisfied. We define the sequences $\left\{z^{(m)}\right\}$ and $\left\{u^{(m)}\right\}$ in the following way:

$$
z^{(0)} \text { is an arbitrary element of } C^{1, L}[c, \varphi, h, Q, \lambda] \text {, }
$$

$$
u^{(0)}=D_{y} z^{(0)} \quad \text { on } I_{c} \text {. }
$$


Evidently, $u^{(0)} \in C^{0, L}\left[c, Q, \lambda_{1}\right]$. Now, if $\left(z^{(m)}, u^{(m)}\right) \in C^{1, L}[c, \varphi, h, Q, \lambda] \times$ $C^{0, L}\left[c, Q, \lambda_{1}\right]$ are known functions, then $u^{(m+1)}$ is a solution of the equation

$$
u=T^{(m)}[u]
$$

and

$$
z^{(m+1)}=F^{(m)}\left[z^{(m)}, u^{(m+1)}\right]
$$

where $T^{(m)}=\left(T_{1}^{(m)}, \ldots, T_{n}^{(m)}\right)$ is defined by

$$
\begin{aligned}
T_{i}^{(m)}[z, u](x, y)= & D_{y_{i}} \varphi(0, g(0 ; x, y)) \\
& -\sum_{l=1}^{r} D_{y_{i}} h_{l}(0, g(0 ; x, y)) z^{(m)}\left(a_{l}, g(0 ; x, y)\right) \\
& -\sum_{l=1}^{r} h_{l}(0, g(0 ; x, y)) u^{(m)}\left(a_{l}, g(0 ; x, y)\right) \\
& +\int_{0}^{x}\left[D_{y_{i}} f\left(P\left[z^{(m)}, u\right](s ; x, y)\right)\right. \\
& \left.+D_{w} f\left(P\left[z^{(m)}, u\right](s ; x, y)\right)\left(u_{i}^{(m)}\right)_{(s, g(s ; x, y))}\right] d s,
\end{aligned}
$$

$i=1, \ldots, n$. We denote by $g$ the solution of

$$
g(t ; x, y)=y+\int_{t}^{x} D_{q} f\left(P\left[z^{(m)}, u\right](\tau ; x, y)\right) d \tau,
$$

and for simplicity we ignore the dependence of $g$ on $z^{(m)}$ and $u$.

Note that the equations $u=T^{(m)}\left[z^{(m)}, u\right]$ and (12) are not identical.

Lemma 2. If Assumptions $\mathrm{H}_{1}-\mathrm{H}_{2}$ are satisfied and if $h_{l} \in C^{1, L}[\bar{S}], \varphi \in$ $C^{1, L}[S], Q_{i}>S_{i}, i=0,1,2$, then there is $c \in(0, a]$ such that for any $m \geq 0$ we have

$\left(a_{m}\right) z^{(m)} \in C^{1, L}[c, \varphi, h, Q, \lambda], u^{(m)} \in C^{0, L}\left[c, Q, \lambda_{1}\right]$

$\left(b_{m}\right) D_{y} z^{(m)}(x, y)=u^{(m)}(x, y)$ in $I_{c}$.

P r o of. We first suppose that $c \in(0, a]$ is a small constant such that

$$
\begin{gathered}
G(c)\left[\bar{S}_{0}+\int_{0}^{c} \alpha\left(s, Q^{\prime}\right) d s\right]<1 \\
{\left[1-\bar{S}_{0}-\int_{0}^{c} \alpha\left(s, Q^{\prime}\right) d s\right]^{-1}\left[S_{1}+\bar{S}_{1} Q_{0}+\int_{0}^{c} \alpha\left(s, Q^{\prime}\right) d s\right] \leq Q_{1},} \\
\left\{1-G(c)\left[\bar{S}_{0}+\int_{0}^{c} \alpha\left(s, Q^{\prime}\right) d s\right]\right\}^{-1} P(c) \leq Q_{2}, \quad M(c)<1, \\
\left(1-\bar{S}_{0}\right)^{-1}\left\{S_{0}+\int_{0}^{c}\left[\beta\left(s, Q_{0}\right)+Q_{1} \alpha\left(s, Q^{\prime}\right)\right] d s\right\} \leq Q_{0},
\end{gathered}
$$

where 


$$
\begin{aligned}
P(x)= & G(x)\left\{S_{2}+\bar{S}_{1} Q_{1}+\bar{S}_{2} Q_{0}+\bar{S}_{1} Q_{1}+\bar{Q}\left(1+Q_{1}\right) \int_{0}^{x} \gamma\left(s, Q^{*}\right) d s\right\} \\
M(x)= & \left\{G(c) Q_{2}\left[\bar{S}_{0}+\int_{0}^{x} \alpha\left(s, Q^{\prime}\right) d s\right]+P(x)\right\} \exp \left[\int_{0}^{x} \gamma\left(s, Q^{*}\right) d s\right] \\
& +\bar{S}_{0}+\left(1+Q_{1}\right) \int_{0}^{x} \gamma\left(s, Q^{*}\right) d s .
\end{aligned}
$$

Put

$$
\begin{aligned}
\lambda_{1}(x)= & \left\{G(c) Q_{2}\left[\bar{S}_{0}+\int_{0}^{c} \alpha\left(s, Q^{\prime}\right) d s\right]+P(c)+1+Q_{1}\right\} \alpha\left(x, Q^{\prime}\right), \\
\lambda_{0}(x)= & \beta\left(x, Q_{0}\right)+\left\{Q_{1}+G(c)\left[S_{1}+\bar{S}_{1} Q_{0}+\bar{S}_{0} Q_{1}\right.\right. \\
& \left.\left.+\bar{Q} \int_{0}^{c}\left[\alpha\left(s, Q^{\prime}\right)+Q_{1} \gamma\left(s, Q^{*}\right)\right] d s\right]\right\} \alpha\left(x, Q^{\prime}\right) .
\end{aligned}
$$

We prove $\left(a_{m}\right)$ and $\left(b_{m}\right)$ by induction. It follows from the definitions of $z^{(0)}$ and $u^{(0)}$ that $\left(a_{0}\right)$ and $\left(b_{0}\right)$ are satisfied. Suppose now that $\left(a_{m}\right)$ and $\left(b_{m}\right)$ hold. First we prove that

$$
T^{(m)}: C^{0, L}\left[c, Q, \lambda_{1}\right] \rightarrow C^{0, L}\left[c, Q, \lambda_{1}\right] .
$$

Indeed, it follows from Assumptions $\mathrm{H}_{1}-\mathrm{H}_{2}$ and Lemma 1 that for $u \in$ $C^{0, L}\left[c, Q, \lambda_{1}\right]$ and $(x, y),(\bar{x}, \bar{y}) \in I_{c}$, we have

$$
\left\|T^{(m)}[u](x, y)\right\| \leq S_{1}+\bar{S}_{1} Q_{0}+\bar{S}_{0} Q_{1}+\left(1+Q_{1}\right) \int_{0}^{x} \alpha\left(s, Q^{\prime}\right) d s \leq Q_{1},
$$

and

$$
\begin{aligned}
\| T^{(m)}[u] & (x, y)-T^{(m)}[u](\bar{x}, \bar{y}) \| \\
\leq & \left\|D_{y} \varphi(0, g(0 ; x, y))-D_{y} \varphi(0, g(0 ; \bar{x}, \bar{y}))\right\| \\
& +\sum_{l=1}^{r}\left\|D_{y} h_{l}(0, g(0 ; x, y))\right\| \cdot\left|z^{(m)}\left(a_{l}, g(0 ; x, y)\right)-z^{(m)}\left(a_{l}, g(0 ; \bar{x}, \bar{y})\right)\right| \\
& +\sum_{l=1}^{r}\left\|D_{y} h_{l}(0, g(0 ; x, y))-D_{y} h_{l}(0, g(0 ; \bar{x}, \bar{y}))\right\| \cdot\left|z^{(m)}\left(a_{l}, g(0 ; \bar{x}, \bar{y})\right)\right| \\
& +\sum_{l=1}^{r} \sum_{k=1}^{n}\left|h_{l}(0, g(0 ; x, y))\right| \cdot\left|u_{k}^{(m)}\left(a_{l}, g(0 ; x, y)\right)-u_{k}^{(m)}\left(a_{l}, g(0 ; \bar{x}, \bar{y})\right)\right| \\
& +\sum_{l=1}^{r} \sum_{k=1}^{n}\left|h_{l}(0, g(0 ; x, y))-h_{l}(0, g(0 ; \bar{x}, \bar{y}))\right| \cdot\left|u_{k}^{(m)}\left(a_{l}, g(0 ; \bar{x}, \bar{y})\right)\right| \\
& +\int_{0}^{x}\left\|D_{y} f\left(P\left[z^{(m)}, u\right](s ; x, y)\right)-D_{y} f\left(P\left[z^{(m)}, u\right](s ; \bar{x}, \bar{y})\right)\right\| d s
\end{aligned}
$$




$$
\begin{aligned}
& +\left|\int_{0}^{x}\left\|D_{y} f\left(P\left[z^{(m)}, u\right](s ; \bar{x}, \bar{y})\right)\right\| d s\right| \\
& +\left|\int_{x}^{\bar{x}} \sum_{k=1}^{n} D_{w} f\left(P\left[z^{(m)}, u\right](s ; \bar{x}, \bar{y})\right)\left(u_{k}^{(m)}\right)_{(s, g(s ; x, y))} d s\right| \\
& \quad+\int_{0}^{x} \sum_{k=1}^{n} \mid D_{w} f\left(P\left[z^{(m)}, u\right](s ; x, y)\right)\left(u_{k}^{(m)}\right)_{(s, g(s ; x, y))} \\
& \quad-D_{w} f\left(P\left[z^{(m)}, u\right](s ; \bar{x}, \bar{y})\right)\left(u_{k}^{(m)}\right)_{(s, g(s ; \bar{x}, \bar{y}))} \mid d s \\
& \leq\left|\int_{x}^{x} \lambda_{1}(t) d t\right|+Q_{2}\|y-\bar{y}\| .
\end{aligned}
$$

By the above inequality and by (16) we get (15).

From Assumptions $\mathrm{H}_{1}-\mathrm{H}_{2}$, applying Lemma 1, we obtain

$$
\begin{aligned}
\| T^{(m)}[u] & (x, y)-T^{(m)}[\bar{u}](x, y) \| \\
\leq & \left\|D_{y} \varphi(0, g(0 ; x, y))-D_{y} \varphi(0, \bar{g}(0 ; x, y))\right\| \\
& +\sum_{l=1}^{r}\left\|D_{y} h_{l}(0, g(0 ; x, y))\right\| \cdot\left|z^{(m)}\left(a_{l}, g(0 ; x, y)\right)-z^{(m)}\left(a_{l}, \bar{g}(0 ; x, y)\right)\right| \\
& +\sum_{l=1}^{r}\left\|D_{y} h_{l}(0, g(0 ; x, y))-D_{y} h_{l}(0, \bar{g}(0 ; x, y))\right\| \cdot\left|z^{(m)}\left(a_{l}, \bar{g}(0 ; x, y)\right)\right| \\
& +\sum_{l=1}^{r} \sum_{k=1}^{n}\left|h_{l}(0, g(0 ; x, y))\right| \cdot\left|u_{k}^{(m)}\left(a_{l}, g(0 ; x, y)\right)-u_{k}^{(m)}\left(a_{l}, \bar{g}(0 ; x, y)\right)\right| \\
& +\sum_{l=1}^{r} \sum_{k=1}^{n}\left|h_{l}(0, g(0 ; x, y))\right| \cdot\left|u_{k}^{(m)}\left(a_{l}, g(0 ; x, y)\right)-\bar{u}_{k}^{(m)}\left(a_{l}, g(0 ; x, y)\right)\right| \\
& +\sum_{l=1}^{r} \sum_{k=1}^{n}\left|h_{l}(0, g(0 ; x, y))-h_{l}(0, \bar{g}(0 ; x, y))\right| \cdot\left|u_{k}^{(m)}\left(a_{l}, \bar{g}(0 ; x, y)\right)\right| \\
& +\int_{0}^{x}\left\|D_{y} f\left(P\left[z^{(m)}, u\right](s ; x, y)\right)-D_{y} f\left(P\left[z^{(m)}, \bar{u}\right](s ; x, y)\right)\right\| d s \\
& +\int_{0}^{x} \sum_{k=1}^{n} \mid D_{w} f\left(P\left[z^{(m)}, u\right](s ; x, y)\right) \\
& -D_{w} f\left(P\left[z^{(m)}, \bar{u}\right](s ; x, y)\right)\left(\bar{u}_{k}^{(m)}\right)_{(s, \bar{g}(s ; x, y)) \mid d s} \\
\leq & M(c)\|u-\bar{u}\|_{C\left(I_{c}, \mathbb{R}^{n}\right)},
\end{aligned}
$$

where $\bar{g}$ is the solution of equation (14) with $\bar{u}$ instead of $u$ on the right-hand side. 
Hence, using the Banach fixed point theorem we conclude that there is exactly one $u^{(m+1)} \in C^{0, L}\left[c, Q, \lambda_{1}\right]$ satisfying (12).

Now we prove that the function $z^{(m+1)}$ given by $(13)$ satisfies $\left(b_{m+1}\right)$. Put

$$
\Delta(x, y, \bar{y})=z^{(m+1)}(x, \bar{y})-z^{(m+1)}(x, y)-\sum_{k=1}^{n} u_{k}^{(m+1)}(x, y)\left(\bar{y}_{k}-y_{k}\right) .
$$

Similarly to [5], [18] we can prove the estimate

$$
\begin{aligned}
|\Delta(x, y, \bar{y})| \leq & \left(S_{2}+\bar{S}_{2} Q_{0}+\bar{S}_{0} Q_{2}\right)\|g(0 ; x, \bar{y})-g(0 ; x, y)\|^{2} \\
& +\int_{0}^{x}\|g(s ; x, \bar{y})-g(s ; x, y)\|^{2}\left[\bar{Q}\left(1+Q_{1}+Q_{2}\right) \gamma\left(s, Q^{*}\right)\right. \\
& \left.+Q_{2} \alpha_{1}\left(s, Q^{\prime}\right)\right] d s .
\end{aligned}
$$

It follows from Lemma 1 that

$$
\|g(s ; x, \bar{y})-g(s ; x, y)\| \leq G(c)\|y-\bar{y}\| .
$$

Therefore there is $C^{*}>0$ such that

$$
|\Delta(x, y, \bar{y})| \leq C^{*}\|y-\bar{y}\|^{2}, \quad(x, y),(x, \bar{y}) \in I_{c} .
$$

Consequently,

$$
D_{y} z^{(m+1)}(x, y)=u^{(m+1)}(x, y), \quad(x, y) \in I_{c} .
$$

It is easily shown that $z^{(m+1)} \in C^{1, L}[c, \varphi, h, Q, \lambda]$. The proof of Lemma 2 is complete.

5. Uniform convergence. We can now formulate the result on the convergence of the sequence of successive approximations.

Lemma 3. If Assumptions $\mathrm{H}_{1}-\mathrm{H}_{2}$ are satisfied and if $h_{l} \in C^{1, L}[\bar{S}], \varphi \in$ $C^{1, L}[S], Q_{i}>S_{i}, i=0,1,2$, then there is $c \in(0, a]$ such that the sequences $\left\{z^{(m)}\right\}$ and $\left\{u^{(m)}\right\}$ are uniformly convergent on $I_{c}$.

Proof. Suppose that $c \in(0, a]$ is a small constant such that

$$
\left[\bar{S}_{1}+\bar{S}_{0}+\int_{0}^{c} H_{1}(s) d s\right] \exp \left\{\int_{0}^{c} H_{0}(s) d s\right\}+\int_{0}^{c} \widetilde{H}_{1}(s) d s<1,
$$

where

$$
\begin{aligned}
& H_{0}(x)=\left\{1+Q_{1}+G(c) Q_{2}\left[\bar{S}_{0}+\int_{0}^{c} \alpha\left(s, Q^{\prime}\right) d s\right]+P(x)\right\} \gamma\left(x, Q^{*}\right), \\
& H_{1}(x)=H_{0}(x)+\alpha\left(x, Q^{\prime}\right), \\
& \widetilde{H}_{i}(x)=K_{0}(x)+\alpha\left(x, Q^{\prime}\right)+K_{1}(c) H_{i}(x), \quad i=1,2,
\end{aligned}
$$




$$
\begin{aligned}
K_{0}(x)= & \gamma\left(x, Q^{*}\right)\left\{Q_{2}+G(c)\left(S_{1}+\bar{S}_{0} Q_{1}+\bar{S}_{2} Q_{0}\right.\right. \\
& \left.\left.+\bar{Q} \int_{0}^{c}\left[\alpha\left(s, Q^{\prime}\right)+Q_{1} \gamma\left(s, Q^{*}\right)\right] d s\right)\right\}, \\
K_{1}(x)= & \exp \left[\int_{0}^{x} H_{0}(s) d s\right] \int_{0}^{x}\left[K_{0}(s)+2 \alpha\left(s, Q^{\prime}\right)\right] d s .
\end{aligned}
$$

For $t \in[0, c]$ and $m \geq 1$ we put

$$
\begin{aligned}
& Z^{(m)}(t)=\sup \left\{\left|z^{(m)}(x, y)-z^{(m-1)}(x, y)\right|:(x, y) \in I_{t}\right\}, \\
& U^{(m)}(t)=\sup \left\{\left\|u^{(m)}(x, y)-u^{(m-1)}(x, y)\right\|:(x, y) \in I_{t}\right\} .
\end{aligned}
$$

According to (12) and Assumptions $\mathrm{H}_{1}, \mathrm{H}_{2}$, on $I_{c}$ we have

$$
\begin{aligned}
\left|u_{i}^{(m+1)}(x, y)-u_{i}^{(m)}(x, y)\right| \leq & \int_{0}^{x} H_{0}(s) U^{(m+1)}(s) d s \\
& +\int_{0}^{x}\left[H_{0}(s) Z^{(m)}(s)+H_{1}(s) U^{(m)}\right] d s \\
& +\bar{S}_{1} Z^{(m)}(x)+\bar{S}_{0} U^{(m)}(x) .
\end{aligned}
$$

Then we obtain the following integral inequality on $[0, c]$ :

$$
\begin{aligned}
U^{(m+1)}(x) \leq & \int_{0}^{x} H_{0}(s) U^{(m+1)}(s) d s+\int_{0}^{x}\left[H_{0}(s) Z^{(m)}(s)+H_{1}(s) U^{(m)}\right] d s \\
& +\bar{S}_{1} Z^{(m)}(x)+\bar{S}_{0} U^{(m)}(x) .
\end{aligned}
$$

The above estimate and the Gronwall lemma imply

$$
\begin{aligned}
U^{(m+1)}(x) \leq & \left\{\int_{0}^{x}\left[H_{0}(s) Z^{(m)}(s)+H_{1}(s) U^{(m)}\right] d s\right. \\
& \left.+\bar{S}_{1} Z^{(m)}(x)+\bar{S}_{0} U^{(m)}(x)\right\} \exp \left\{\int_{0}^{x} H_{0}(s) d s\right\} .
\end{aligned}
$$

Using (13) and Assumptions $\mathrm{H}_{1}, \mathrm{H}_{2}$ we can prove that

$$
\begin{aligned}
Z^{(m+1)}(x) \leq & \bar{S}_{0} Z^{(m)}(x) \\
& +\int_{0}^{x}\left[\widetilde{H}_{0}(s) Z^{(m)}(s)+\widetilde{H}_{1}(s) U^{(m)}(s)\right] d s, \quad x \in[0, c] .
\end{aligned}
$$

From (17), (18) we conclude that there exists $\delta \in(0,1)$ such that

$$
U^{(m+1)}(x)+Z^{(m+1)}(x) \leq \delta\left[U^{(m)}(x)+Z^{(m)}(x)\right], \quad x \in[0, c], m \geq 0 .
$$


From (11) and from Assumptions $\mathrm{H}_{1}, \mathrm{H}_{2}$ we have

$$
\begin{aligned}
& Z^{(1)}(x) \leq \int_{0}^{x}\left[\beta\left(s, Q_{0}\right)+\widetilde{Q}_{1} \alpha\left(s, Q^{\prime}\right)\right] d s, \\
& U^{(1)}(x) \leq\left(1+\widetilde{Q}_{1}\right) \int_{0}^{x} \alpha\left(s, Q^{\prime}\right) d s .
\end{aligned}
$$

Finally, the assertion of Lemma 3 follows from (19).

6. Theorem and particular cases. We are now able to state the main result of the paper.

Theorem. Let Assumptions $\mathrm{H}_{1}-\mathrm{H}_{2}$ hold, and let $Q_{i}>S_{i}, i=0,1,2$. Then there is $c \in(0, a]$ such that for any numbers $a_{l}, 0 \leq a_{l} \leq c, l=$ $1, \ldots, r$, and functions $\varphi \in C^{1, L}[S], h_{l} \in C^{1, L}[\bar{S}]$, there exists a solution $\bar{z}: E_{c} \rightarrow \mathbb{R}$ of problem (1), (2).

Proof. By Lemma 3 there exist $\bar{z} \in C^{1, L}[c, \varphi, h, Q, \lambda]$ and $\bar{u} \in$ $C^{0, L}\left[c, Q, \lambda_{1}\right]$ such that $\left\{z^{(m)}\right\}$ converges to $\bar{z}$ and $\left\{u^{(m)}\right\}$ converges to $\bar{u}$ uniformly on $I_{c}$. Furthermore, $D_{y} \bar{z}$ exists on $I_{c}$ and $D_{y} \bar{z}=\bar{u}$. Thus we obtain

$$
\begin{aligned}
\bar{z}(x, y)= & \varphi(0, \bar{g}(0 ; x, y))-\sum_{l=1}^{r} h_{l}(0, \bar{g}(0 ; x, y)) \bar{z}\left(a_{l}, \bar{g}(0 ; x, y)\right) \\
& +\int_{0}^{x}\left[f\left(P\left[\bar{z}, D_{y} \bar{z}\right](s ; x, y)\right)\right. \\
& \left.-\sum_{k=1}^{n} D_{q_{k}} f\left(P\left[\bar{z}, D_{y} \bar{z}\right](s ; x, y)\right) D_{y_{k}} \bar{z}(s ; x, y)\right] d s
\end{aligned}
$$

where

$$
\bar{g}(t ; x, y)=y+\int_{t}^{x} D_{q} f\left(P\left[\bar{z}, D_{y} \bar{z}\right](\tau ; x, y)\right) d \tau .
$$

Now we prove that $\bar{z}$ is a solution of (1). By taking $y=\bar{g}(x ; 0, \eta)$ in $(20)$, we get

$$
\begin{aligned}
& (21) \quad \bar{z}(x, \bar{g}(x ; 0, \eta)) \\
& =\varphi(0, \eta)-\sum_{l=1}^{r} h_{l}(0, \eta) \bar{z}\left(a_{l}, \eta\right) \\
& +\int_{0}^{x}\left[f\left(\tau, \bar{g}(x ; 0, \eta), \bar{z}_{(\tau, \bar{g}(x ; 0, \eta))}, D_{y} \bar{z}(\tau, \bar{g}(x ; 0, \eta))\right)\right. \\
& \left.-\sum_{k=1}^{n} D_{q_{k}} f\left(\tau, \bar{g}(x ; 0, \eta), \bar{z}_{(\tau, \bar{g}(x ; 0, \eta))}, D_{y} \bar{z}(\tau, \bar{g}(x ; 0, \eta))\right) D_{y_{k}} \bar{z}(\tau, \bar{g}(x ; 0, \eta))\right] d \tau
\end{aligned}
$$


since the function $\bar{g}$ satisfies the group property. By differentiation of (21) with respect to $x$, and by putting again $y=\bar{g}(x ; 0, \eta)$, we find that $\bar{z}$ satisfies (1). It follows immediately that $\bar{z}$ satisfies (2). The proof of the Theorem is complete.

Now we list examples of equations which can be derived from (1).

1) Suppose that $\bar{f}: I_{a} \times \mathbb{R} \times \mathbb{R}^{n} \rightarrow \mathbb{R}, \lambda:[0, a] \rightarrow \mathbb{R}, \psi: I_{a} \rightarrow \mathbb{R}^{n}$, are given. Assume that

$$
-b_{0} \leq \lambda(x)-x \leq 0, \quad-b \leq \psi(x, y)-y \leq b, \quad(x, y) \in I_{a} .
$$

We consider the operator $f$ defined by

$$
f(x, y, w, q)=\bar{f}(x, y, w[\lambda(x)-x, \psi(x, y)-y], q), \quad(x, y, w, q) \in \Omega^{(0)} .
$$

In this case equation (1) reduces to the following differential equation with a retarded argument:

$$
\begin{aligned}
D_{x} z(x, y) & =\bar{f}\left(x, y, z(\lambda(x), \psi(x, y)), D_{y} z(x, y)\right), \\
z(x, y) & =\varphi(x, y), \quad(x, y) \in E_{0} .
\end{aligned}
$$

2) Suppose that $\bar{f}: I_{a} \times \mathbb{R} \times \mathbb{R}^{n} \rightarrow \mathbb{R}$ is given function. Let

$$
f(x, y, w, q)=\bar{f}\left(x, y, \int_{B} w(t, s) d t d s, q\right) .
$$

Then equation (1) reduces to the differential-integral equation

$$
\begin{aligned}
D_{x} z(x, y) & =\bar{f}\left(x, y, \int_{-b_{0}}^{0} \int_{-b}^{b} z(x+t, y+s) d t d s, D_{y} z(x, y)\right), \\
z(x, y) & =\varphi(x, y), \quad(x, y) \in E_{0} .
\end{aligned}
$$

3) If we take

$$
f(x, y, w, q)=\bar{f}\left(x, y,\left(V\left(I_{(x, y)} w\right)\right)(x, y)\right),
$$

where $\left(I_{(x, y)} w\right)(t, s)=w(t-x, s-y)$, then equation (1) reduces to the differential-functional equation [25]

$$
D_{x} z(x, y)=\bar{f}\left(x, y,(V z)(x, y), D_{y} z(x, y)\right) .
$$

\section{References}

[1] P. Bassanini, Su una recente dimostrazione cirza il problema di Cauchy per sistemi quasi lineari iperbolici, Boll. Un. Mat. Ital. B (5) 13 (1976), 322-335.

[2] - - On a recent proof concerning a boundary value problem for quasilinear hyperbolic systems in the Schauder canonic form, ibid. A (5) 14 (1977), 325-332.

[3] - Iterative methods for quasilinear hyperbolic systems, ibid. B (6) 1 (1982), 225250 . 
[4] P. Bassanini e M. C. Salvatori, Un problema ai limiti per sistemi integrodifferenziali non lineari di tipo iperbolico, ibid. B (5) 18 (1981), 785-798.

[5] P. Brandi, Z. Kamont and A. Salvadori, Existence of weak solutions for partial differential-functional equations, preprint.

[6] L. Byszewski, Existence and uniqueness of solutions of nonlocal problems for hyperbolic equation $u_{x t}=F\left(x, t, u, u_{x}\right)$, J. Appl. Math. Stochastic Anal. 3 (1990), $163-168$.

[7] —, Theorem about existence and uniqueness of continuous solution of nonlocal problem for nonlinear hyperbolic equation, Appl. Anal. 40 (1991), 173-180.

[8] _, Existence of a solution of a Fourier nonlocal quasilinear parabolic problem, J. Appl. Math. Stochastic Anal. 5 (1992), 43-68.

[9] - Monotone iterative method for a system of nonlocal initial-boundary parabolic problems, J. Math. Anal. Appl. 177 (1993), 445-458.

[10] L. Byszewski and V. Lakshmikantham, Monotone iterative technique for nonlocal hyperbolic differential problem, J. Math. Phys. Sci. 26 (1992), 346-359.

[11] L. Cesari, A boundary value problem for quasilinear hyperbolic system, Riv. Mat. Univ. Parma 3 (1974), 107-131.

[12] -, A boundary value problem for quasilinear hyperbolic system in the Schauder canonic form, Ann. Scuola Norm. Sup. Pisa (4) 1 (1974), 311-358.

[13] J. Chabrowski, On non-local problems for parabolic equations, Nagoya Math. J. 93 (1984), 109-131.

[14] M. Cinquini-Cibrario e S. Cinquini, Equazioni alle derivate parziali di tipo iperbolico, Cremonese, Roma, 1964.

[15] T. Człapiński, On existence and uniqueness of solutions of nonlocal problems for hyperbolic differential-functional equations, preprint.

[16] - On the Cauchy problem for quasilinear hyperbolic systems of partial differentialfunctional equations of the first order, Z. Anal. Anwendungen 10 (1991), 169-182.

[17] T. Człapiński and Z. Kamont, Generalized solutions of quasi-linear hyperbolic systems of partial differential-functional equations, J. Math. Anal. Appl. 172 (1993), 353-370.

[18] Z. Kamont, Hyperbolic Functional Differential Inequalities and Applications, Klwer, 1999, to appear.

[19] Z. Kamont and J. Turo, On the Cauchy problem for quasilinear hyperbolic system of partial differential equations with a retarded argument, Boll. Un. Mat. Ital. B (6) 4 (1985), 901-916.

[20] - , - A boundary value problem for quasilinear hyperbolic systems with a retarded argument, Ann. Polon. Math. 47 (1987), 347-360.

[21] A. Pliś, Generalization of the Cauchy problem for a system of partial differential equations, Bull. Acad. Polon. Sci. Cl. III 4 (1956), 741-744.

[22] P. Pucci, Problemi ai limiti per sistemi di equazioni iperboliche, Boll. Un. Mat. Ital. B (5) 16 (1979), 87-99.

[23] J. Szarski, Generalized Cauchy problem for differential-functional equations with first order partial derivatives, Bull. Acad. Polon. Sci. Sér. Sci. Math. Astronom. Phys. 24 (1976), 575-580.

[24] J. Turo, On some class of quasilinear hyperbolic systems of partial differentialfunctional equations of the first order, Czechoslovak Math. J. 36 (1986), 185-197.

[25] - Generalized solutions of the Cauchy problem for nonlinear functional partial differential equations, Z. Anal. Anwendungen 7 (1988), 127-133.

[26] - Generalized solutions to functional partial differential equations of the first order, Zeszyty Nauk. Politech. Gdańskiej Mat. 14 (1988), 1-99. 
[27] J. Turo, A boundary value problem for hyperbolic systems of differential-functional equations, Nonlinear Anal. 13 (1989), 7-18.

[28] —, Classical solutions to nonlinear hyperbolic functional partial differential equations, An. Ştiinţ. Univ. Al. I. Cuza Iaşi Mat. (N.S.), to appear.

Department of Mathematics

Technical University of Gdańsk

Narutowicza 11/12

80-952 Gdańsk, Poland

E-mail: turo@mif.pg.gda.pl

Reçu par la Rédaction le 11.12.1997

Révisé le 15.4.1999 